\title{
Analysis of Spectral Amplitude Coding OCDMA Successive Interference Cancellation scheme
}

\author{
Tawfig Eltaif ${ }^{1 *}$, Hossam M. H. Shalaby ${ }^{2}$, Sahbudin Shaari ${ }^{1}$, Mohammad M. N. Hamarsheh ${ }^{3}$ \\ ${ }^{1}$ Photonics Technology Laboratory (PTL), Institute of Micro Engineering and Nanoelectronics, \\ University Kebangsaan Malaysia, 43600 UKM Bangi, Selangor, MALAYSIA \\ ${ }^{2}$ Elect. Eng. Dept, Fac. of Engineering, Alexandria University, Alexandria 21544, EGYPT. \\ ${ }^{3}$ Dept. of Telecommunication Engineering (TCE), Fac. of Information Technology, Arab American University, \\ Jenin, P.O. BOX 1: 240 Jenin, P.O. Box 2: 13 Zababdeh, PALESTINE \\ *tefosat@ieee.org
}

\begin{abstract}
A successive interference cancellation optical (O-CDMA) system with spectral amplitude coding is investigated. It has been shown that SIC/SAC scheme has potential to suppress MAI, and improve the system performance as compared with conventional receiver.
\end{abstract}

Keywords: OCDMA, successive interference cancellation (SIC).

\section{INTRODUCTION}

Spectral-amplitude-coding (SAC) OCDMA system was first investigated by Kavehrad and Zaccarin in 1995 [1]. It is shown that the system can cancel multiple access interference (MAI) by using code sequences with fixed in phase cross correlation, but the phase-induced intensity noise (PIIN) acts as the main reason limits significantly the system performance. We have proposed the successive interference cancellation (SIC) scheme to suppress MAI in optical CDMA systems [2]. It has been shown that, the system with SIC scheme using optical orthogonal codes, and modified prime codes, has much lower bit error rate performance as compared with the one without cancellation [2-3]. In this paper we have analyzed theoretically SIC/SAC optical CDMA using modified frequency hopping (MFH) codes. It has been clearly shown that the system with cancellation scheme has much lower BER performance as compared with the one without cancellation.

\section{DESCRIPTION OF SIC/SAC OPTICAL CDMA}

SIC scheme is applied to SAC optical CDMA systems using MFH codes as shown in Fig.1. The received signal is fed up to $N$ components of SAC. Each bit of data received will be split and detected by the complementary scheme [1]. The main idea of SAC technique that the receiver filters the incoming signal with the same filter (direct decoder, $A(v)$ ) used at the transmitter as well as its complementary filter (complementary decoder, $\bar{A}(v)$ ). The output filters are detected by two photodetectors connected in a balanced fashion. SIC scheme idea is based on the following (i) Recognize the strongest signal (the one with maximum correlation value); (ii) Decode the strongest signal; (iii) Regenerate the strongest signal using its chip sequence; (iv) Subtract the strongest signal; and (v) Repeat until all users are decoded or a permissible number of cancellations are achieved [2-3].

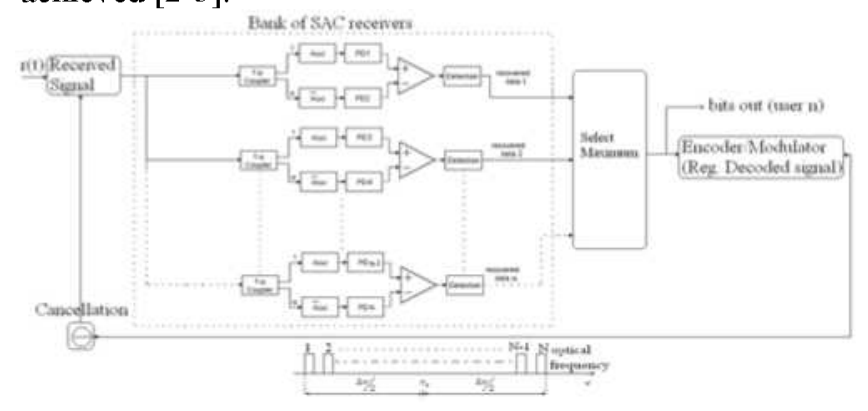

Fig. 1 SIC/SAC receiver scheme

\section{PERFORMANCE ANALYSIS}

In our analysis we have followed the same method as in [4], where the MFH codes have the same properties of MQC codes [5], in MFH code each code sequence has $\left(q^{2}+q\right)$ elements that can be divided into $(q+1)$ groups, and each contain one " 1 " and $(q-1) " 0 s$ ". In our analysis we consider PIIN noise, shot noise, and thermal noise. Detailed analysis of the SIC scheme can be found in [2-3]. In general for the $j^{\text {th }}$ cancellations we get $r_{j}(t)=r_{j-1}(t)-Z_{j} \cdot \sum_{i=1}^{F} c_{j}(i)$, where $Z_{j}$ refers to the correlation after $(j-1)^{\text {th }}$ cancellation, then the decision variable for the $(j+1)$ th user is $Z_{j+1}=\mathfrak{R} \frac{P_{e r}}{q} b_{j+1}+\left\langle i_{j+1}^{2}\right\rangle$, Where $\left\langle i_{j+1}^{2}\right\rangle$ is the interference noise of the desired user included some noise due to imperfect cancellation, this interference expressed in Eq. (1), and SNR is expressed in Eq. (2), where $B$ is the noise-equivalent electrical bandwidth of the receiver, $e$ is the electron's charge, $K_{b}$ is Boltzmann's constant, $T_{n}$ is the absolute receiver noise temperature, $R_{l}$ is the receiver load resistor, $\Delta v$ is the encoded optical bandwidth in Hertz; $N$ is the number of active users. 


$$
\begin{gathered}
\left.\left\langle i_{j+1}^{2}\right\rangle=\frac{e B \Re P_{e r}}{\left(q^{2}+q\right)}\left(2 N+q-1-2 \sum_{i}^{j}\left\langle i_{i}^{2}\right\rangle\right)\right)+\frac{B \Re^{2} N P_{e r}^{2}}{2 q^{2}(q+1) \Delta v}\left[\frac{N-1}{q}+q+N-\frac{1}{N} \sum_{i}^{j}\left\langle i_{i}^{2}\right\rangle\right]+4 K_{b} T_{n} B / R_{L} \\
S N R_{j+1}=\frac{\mathfrak{R}^{2} \frac{P_{e r}^{2}}{q^{2}}}{\left.\frac{e B \Re P_{e r}}{\left(q^{2}+q\right)}\left(2 N+q-1-2 \sum_{i}^{j}\left\langle i_{i}^{2}\right\rangle\right)\right)+\frac{B \mathfrak{R}^{2} N P_{e r}^{2}}{2 q^{2}(q+1) \Delta v}\left[\frac{N-1}{q}+q+N-\frac{1}{N} \sum_{i}^{j}\left\langle i_{i}^{2}\right\rangle\right]+4 K_{b} T_{n} B / R_{L}}
\end{gathered}
$$

The responsivity of the photodetector is given by $\mathfrak{R}=\eta e / h v_{c}$. Here, $\eta$ is the quantum efficiency, $e$ is the electron charge, $h$ is Plank's constant, and $v_{c}$ is the central frequency of the original broadband optical pulse. Therefore, the probability of error (BER) after the $j^{\text {th }}$ cancellation can be estimated using the Gaussian approximation $B E R_{j+1}=Q\left(\sqrt{S N R_{j+1}}\right)$. The typical parameters used in our calculations are, $193.1 \mathrm{THz}$ operating wavelength, 0.6 photodiode quantum efficiency, $80 \mathrm{MHz}$ electrical equivalent bandwidth, $300 \mathrm{k}$ receiver noise temperature, $\Delta v=3.75 \mathrm{THz}$ line-width of the thermal noise, and $1030 \Omega$ receiver load resistor. In Fig. 2 It has been shown that at effective power $P_{e r}=-20 \mathrm{dBm}$. the system with MFH codes has better performance than MQC codes as expected, because the value of $(q=16)$ larger than $(p=13)$. However, the figure also shows that our SIC/SAC receiver scheme (i.e. with cancellation) has better performance than the one without cancellation. Further, Fig. 3 shows the system is affect by the intensity noise, shot noise and thermal noise, when the $P_{e r}=-30 \mathrm{dBm}$, and the main reason of that came from the large number of prime code, which causes a large power loss at the transmitter part. The BER function of optical CDMA systems using modified quadratic congruence codes is plotted in the same Fig. 4 for the sake of comparison. It has been shown that the SIC cancellation scheme has better performance than conventional scheme and SIC/SAC scheme. Assuming that prime number set as a length 7 , it is clearly for similar BER $\left(10^{-9}\right)$, less than 10 users can be active with conventional scheme and SIC/SAC cancellation scheme, which can be increased to 25 users with SIC cancellation scheme.

\section{CONCLUSION}

In this paper, we purposed to mitigate multiple access interference (MAI), and suppress the intensity noise using successive interference cancellation with spectral amplitude coding (SIC/SAC) for optical CDMA system. It has been shown that, the system that uses MFH codes has better a performance (i.e. BER) than the one using MQC codes. Further, the system shows much lower BER performance with SIC/SAC cancellation scheme as compared to the one without cancellation. Furthermore, using SIC optical CDMA system shows much lower BER performance as compared to the one without cancellation and to SIC/SAC cancellation scheme. However, the interference and intensity noise have the main effect of the system at low effective power.

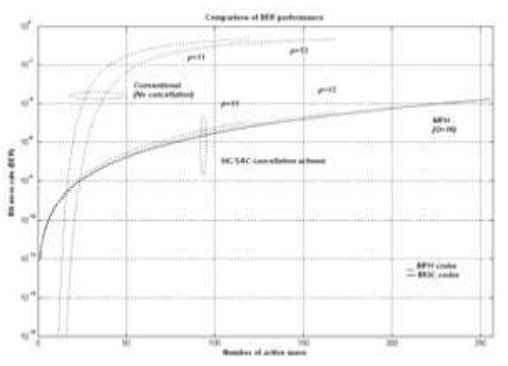

Fig. 2 BER versus number of active users

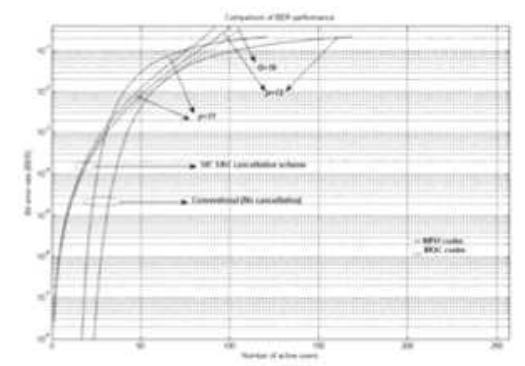

Fig. 3 Comparison of BER at $P_{e r}=-30 \mathrm{dBm}$

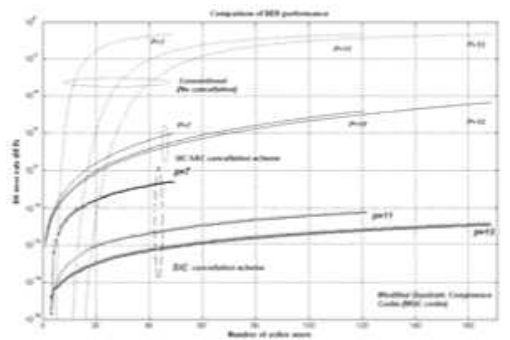

Fig. 4 Comparison of BER performances

\section{REFERENCES}

[1] M. Kavehrad and D. Zaccarin, "Optical code-division-multiplexed systems based on spectral encoding of noncoherent sources," J. Lightw. Technol., vol. 13, no. 3, pp. 534-545, Mar. 1995.

[2] T Eltaif, H M H Shalaby, S Shaari, and M. M. N. Hamarsheh. "A proposal of successive interference cancellation scheme in optical CDMA systems," optic. Eng. SPIE. Vol. 47. no. 3, 2008.

[3] T Eltaif, H M H Shalaby, S Shaari, \& M. M. N. Hamarsheh "Analytical comparison of optical CDMA system with and without successive interference cancellation scheme using modified prime sequence codes," Opt. Eng., Proc. SPIE, vol. 47(9), pp. 095001(1-6), Sept. 2008.

[4] W. Zou, H. M. H. Shalaby, and H. G. Shiraz, "Modified quadratic congruence codes for fiber Bragg-grating-based spectral-amplitude-coding optical CDMA systems," J. Lightwave Technol., vol. LT-19, pp. 1274-1281, Sept. 2001.

[5] Z. Wei, H. M. Shalaby, and H. Ghafouri-Shiraz, "Proposal of a Novel Code for Spectral Amplitude-Coding Optical CDMA Systems," IEEE Photon. Technol. Lett., 14, NO. 3, Mar. 2002. 